\section{Profile of patients treated in dentistry course discipline of Federal University of Espírito Santo, Brazil}

\title{
Perfil dos pacientes atendidos em disciplina do curso de Odontologia da Universidade Federal do Espírito Santo
}

ABSTRACT | Introduction:

The overall population has been using quality and mostly free-of-charge dental care services provided by universities. Objectives: Identifying the sociodemographic profile, health bistory and oral health babits of patients treated in the Dental Restoration Clinic

(ORC) of UFES. Methods: Cross-sectional descriptive study based on dental records. Collected data were subjected to descriptive statistics, Fisher's exact test and Maximum Likelihood Ratio. Results: In total, 222 individuals were treated. Patients in the age group $51-60$ years $(38.8 \%)$, women $(75.2 \%)$ and those living in the metropolitan region of

Vitória $(94.60 \%)$ were the ones who mostly used the investigated services. In total, 150 patients reported to have systemic diseases, $39.3 \%$ of them had more than 3 concomitant diseases. Cardiovascular (23.78\%) and infectious diseases (15.36\%) were the most prevalent systemic changes. Regular use of medication was reported by 132 patients; it was significantly bigher in women (65.9\%) and in the age group 51-60 years $(79.1 \%)(p=0.00)$. Antihypertensive drug using was reported by the largest number of patients (21.72\%), and they were followed by anxiolytics $(17.17 \%)$. With respect to oral hygiene, most patients reported to brush their teeth 3 times a day (63\%), as well as to floss on a daily basis (63.5\%); this habit was mostly prevalent among women $71.3 \%$,

$p=0.00)$. Conclusion: Patients were mostly women, in the age group 51-60 years. Cardiovascular diseases were most often referred, and antibypertensive medications were most often used by participants, who reported high sugar intake and tooth brushing 3 time a day.

Keywords | Dental Care; Public Health; Epidemiology.
RESUMO| Introdução: A população tem buscado o serviço odontológico das Universidades considerado de qualidade e em grande parte gratuito. Objetivo: Identificar o perfil sociodemográfico, histórico de saúde e hábitos de saúde bucal dos pacientes atendidos na disciplina de Odontologia Restauradora Clínica (ORC) da UFES. Métodos: Trata-se de um estudo transversal descritivo a partir de prontuários odontológicos. Os dados coletados foram submetidos à estatística descritiva, teste de Fischer e Razão de Máxima Verossimilhança. Resultados: Foram atendidos 222 indivíduos. Pacientes na faixa etária de 51-60 anos (38,8\%), mulheres (75,2\%) e procedentes da Região Metropolitana de Vitória-ES (94,60\%) foram os que mais buscaram por atendimento. Um total de 150 pacientes relatou possuir doenças sistêmicas, sendo 39,3\% com mais de 3 doenças concomitantes. As alterações sistêmicas mais prevalentes foram as cardiovasculares $(23,78 \%)$ e as doenças infecciosas (15,36\%). O uso regular de medicamentos foi relatado por 132 pacientes, significativamente maior em mulheres $(65,9 \%)$ e na faixa etária de 51-60 anos $(79,1 \%)(p=0,00)$. O uso de anti-hipertensivos foi referido por maior número de pacientes $(21,72 \%)$, seguidos dos ansiolíticos (17,17\%). Quanto aos cuidados com higiene bucal, a maioria dos pacientes relatou escovar os dentes 3x/dia (63\%) e usar diariamente o fio dental $(63,5 \%)$, sendo este mais prevalente nas mulheres $(71,3 \%)(p=0,00)$. Conclusão: Os pacientes foram predominantemente do sexo feminino, na faixa etária de 51 a 60 anos. As doenças cardiovasculares foram mais referidas, sendo os anti-hipertensivos a medicação mais utilizada. Relataram alto consumo de açúcar e escovação dentária $3 \mathrm{x} /$ dia.

Palavras-chave | Assistência Odontológica; Saúde Pública; Epidemiologia.

${ }^{1}$ Universidade Federal do Espírito Santo. Vitória/ES. Brasil. 


\section{INTRODUÇÃO}

Desde a implementação do Programa Brasil Sorridente pelo Ministério da Saúde, o acesso da população à saúde bucal tem melhorado, sendo a primeira política nacional realizada especificamente para a saúde bucal e visa à prevenção, tratamento básico, atendimento especializado e reabilitação oral. A população é atendida nas Unidades Básicas de Saúde (UBS) e nos Centros de Especialidades Odontológicas $(\mathrm{CEOs})^{1}$. Os cuidados preventivos de saúde bucal estão relacionados com a escolaridade, sexo e o perfil socioeconômico do paciente ${ }^{2,3}$. As UBS não conseguem cobrir todas as necessidades da população, pois os serviços oferecidos são voltados para a atenção primária e ainda há um número reduzido de CEOs. Desse modo, uma parte da população de menor renda busca atendimento nas universidades ${ }^{4,5}$.

Ter o conhecimento sobre o perfil e as necessidades dos indivíduos que procuram os serviços prestados nas Universidades e entender suas deficiências e limitações podem ser uma maneira de melhorar a dinâmica de funcionamento das disciplinas clínicas, bem como favorecer a logística de atendimento e reduzir gastos públicos com saúde bucal ${ }^{6}$. Esse conhecimento pode contribuir para a diminuição da quantidade de faltas dos pacientes, pois a sua ausência no horário marcado gera prejuízo no tratamento e na aprendizagem do aluno ${ }^{7,8}$.

São de grande importância os dados sobre a história médica do paciente, pois a saúde geral está relacionada com a saúde bucal, e o uso de alguns medicamentos pode interferir no atendimento odontológico ${ }^{2,4}$. A anamnese do paciente deve ser realizada de forma clara e objetiva. Os dados relacionados a hábitos de saúde bucal também são importantes, pois revelam hábitos deletérios como uso de palito dental, alimentos açucarados, e outros fatores interferentes como a experiência prévia com flúor e escovação dentária, ${ }^{910}$. Todos esses dados são obtidos por meio do Prontuário Odontológico Único (PU), sendo necessário que o paciente forneça as informações de maneira correta sem nenhuma omissão ${ }^{11}$.

O conceito emergente de saúde prospectivo visa mudar o foco de cuidados de gestão da doença para gestão em saúde, ou seja, prevenção de doenças ${ }^{12}$. Os cursos de Odontologia devem preparar melhor os dentistas do futuro para ter sucesso nesse novo ambiente de cuidados de saúde, fornecendo-lhes conhecimentos técnico-científicos para compreender, avaliar o risco de doenças e praticar a prevenção $0^{3,13}$.

O objetivo do presente estudo foi identificar o perfil e o histórico de saúde geral e bucal dos pacientes atendidos na disciplina de Odontologia Restauradora Clínica (ORC) do Curso de Odontologia da Universidade Federal do Espírito Santo (UFES).

\section{MÉTODOS|}

Esta pesquisa foi aprovada pelo Comitê de Ética local, parecer CEP $\mathrm{n}^{\circ}$ 1.544.313. Trata-se de um estudo transversal descritivo a partir de prontuários odontológicos. Foram analisados prontuários de pacientes atendidos por alunos na disciplina de ORC do Curso de Graduação em Odontologia da UFES, no período de 2010 a 2015.

Foram coletados os dados referentes à idade, sexo, presença de doenças, uso regular de medicações e hábitos de saúde bucal. Critérios de inclusão: foram analisados os prontuários registrados no Sistema Informatizado de Ensino (SIE) e direcionados para a disciplina de ORC. Critérios de exclusão: foram excluídos os prontuários de pacientes que não assinaram o termo de autorização para utilização dos dados contidos nos prontuários.

Os dados coletados foram submetidos à estatística descritiva simples (valores absolutos e relativos), teste de Fischer e Razão de Máxima Verossimilhança, sendo estabelecido um intervalo de confiança de $95 \%(\mathrm{p}<0,05)$.

\section{RESULTADOS}

No período em estudo, um total de 385 prontuários foram acessados para a coleta de dados. Destes, 222 foram de pacientes atendidos na disciplina de ORC. Dentre eles, 113 apresentavam tratamentos concluídos.

Verificou-se que a maior procura por atendimento, em todas as faixas etárias, foi de pacientes do sexo feminino. A faixa etária de mais de 50 anos foi a mais frequente (Tabela 1). 
Tabela 1 - Distribuição quanto ao sexo e faixa etária dos pacientes atendidos na disciplina de Odontologia Restauradora Clínica da UFES, no periodo de 2010 a 2015. Vitória/ES, Brasil

\begin{tabular}{|c|c|c|c|c|c|c|}
\hline \multicolumn{7}{|c|}{ SEXO } \\
\hline \multirow[b]{2}{*}{ FAIXA ETÁRIA } & \multicolumn{2}{|c|}{ FEMININO } & \multicolumn{2}{|c|}{ MASCULINO } & \multicolumn{2}{|c|}{ TOTAL } \\
\hline & $\mathbf{n}$ & $\%$ & $n$ & $\%$ & $\mathbf{n}$ & $\%$ \\
\hline $21-30$ anos & 29 & 13,1 & 13 & 5,9 & 42 & 18,9 \\
\hline $31-40$ anos & 42 & 18,9 & 8 & 3,6 & 50 & 22,5 \\
\hline $41-50$ anos & 34 & 15,3 & 10 & 4,5 & 44 & 19,8 \\
\hline $51-60$ anos & 62 & 27,9 & 24 & 10,8 & 86 & 38,8 \\
\hline TOTAL & 167 & 75,2 & 55 & 24,8 & 222 & 100,0 \\
\hline
\end{tabular}

A maioria dos pacientes (94,6\%) era procedente da Região Metropolitana de Vitória, que compreende os municípios de Cariacica, Fundão, Guarapari, Serra, Viana, Vila Velha e a capital, Vitória. Um total de 45 pacientes (20,3\%) reside na capital, e apenas 12 pacientes (5,4\%) vieram de cidades do interior do estado do Espírito Santo.

Observou-se que 150 pacientes $(67,6 \%)$ possuíam alguma doença sistêmica, sendo que a maioria deles (39,3\%) afirmou ter três ou mais doenças concomitantes. Não foi observada diferença significativa quanto à presença e número de doença entre os sexos. A faixa etária entre 51 a 60 anos apresentou a maior prevalência de doenças sistêmicas, sendo a diferença estatisticamente significativa em relação às demais faixas etárias $(\mathrm{p}=0,00)$. Quanto ao número de doenças relatadas por pacientes, não houve diferença estatística de acordo com a idade $(\mathrm{p}=0,20)$ (Tabela 2).

Tabela 2 - Distribuição de pacientes com doenças sistêmicas e do número de doenças por pacientes atendidos na disciplina de Odontologia Restauradora Clínica da UFES, no período de 2010 a 2015. Vitória/ES, Brasil

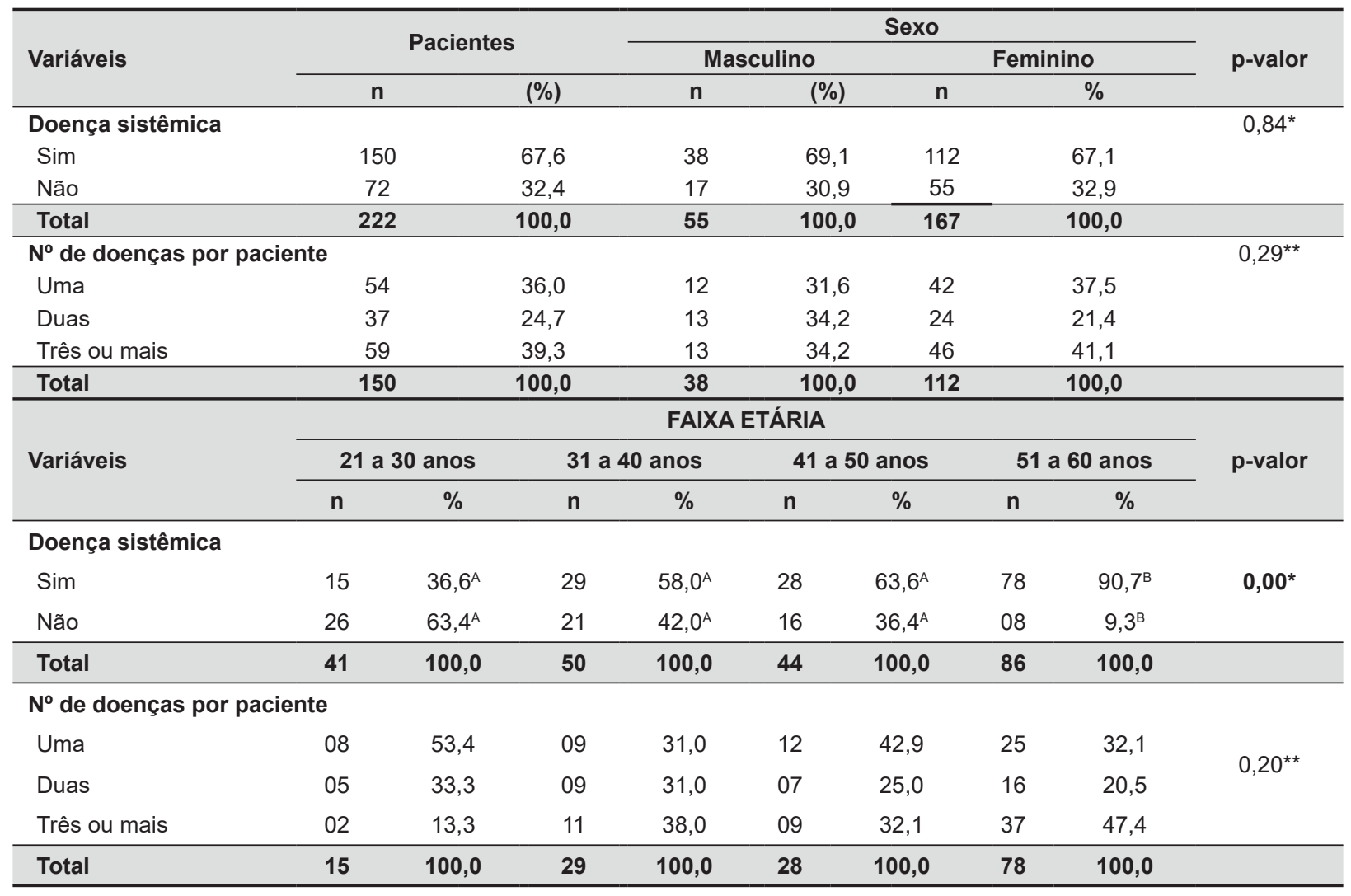

* Teste exato de Fisher. ** Razão de Máxima Verossimilhança. Diferença significativa para p <0,05. Letras diferentes denotam diferenças estatisticamente significantes entre as proporções. 
As alterações sistêmicas mais prevalentes foram as cardiovasculares (23,8\%), seguidas das doenças infecciosas $(15,4 \%)$, doenças gastrointestinais $(12,8 \%)$, problemas respiratórios $(11,3 \%)$, alergias $(10,1 \%)$, alterações endócrinas $(7,8 \%)$ e alterações neurológicas (4,9\%). Artrite reumatoide, câncer de colo de útero, glaucoma, lúpus eritematoso sistêmico e osteoporose corresponderam juntos a $13,9 \%$ das enfermidades relatadas.

O uso regular de medicamentos foi significativamente maior no sexo feminino $(p=0,00)$. A faixa etária entre 51 a 60 anos apresentou um maior uso de medicamentos, sendo a diferença estatisticamente significativa em relação às demais faixas etárias $(p=0,00)$, como pode ser observado na Tabela 3 .

Dentre os medicamentos de uso regular, os antihipertensivos agruparam o maior número de pacientes (43 indivíduos, 21,7\%), seguido dos ansiolíticos e antidepressivos, 34 pacientes $(17,2 \%)$, enquanto o uso de anticoncepcional foi relatado por 25 pacientes (12,6\%). Outros medicamentos incluíram os imunossupressores, reposição de cálcio, relaxantes musculares, anticoagulantes, dentre outros, e juntos corresponderam a $14,6 \%$ das medicações relatadas (Gráfico 1).

Tabela 3 - Distribuição do uso regular de medicamentos quanto sexo e faixa etária dos pacientes atendidos na disciplina de Odontologia Restauradora Clínica da UFES, no período de 2010 a 2015. Vitória/ES, Brasil

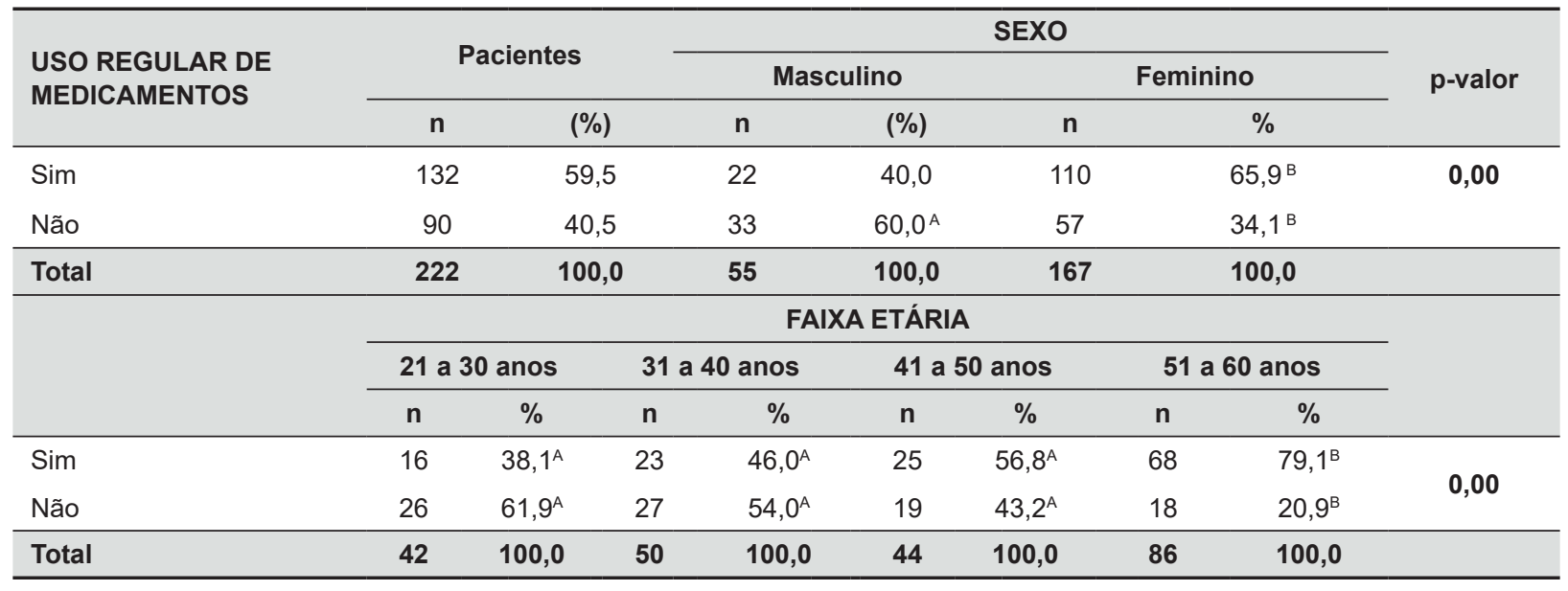

Teste exato de Fisher. Diferença significativa para $\mathrm{p}<0,05$. Letras diferentes denotam diferenças estatisticamente significantes entre as proporções.

Gráfico 1 - Distribuição da medicação de uso regular dos pacientes atendidos na disciplina de Odontologia Restauradora Clínica da UFES, no periodo de 2010 a 2015. Vitória/ES, Brasil

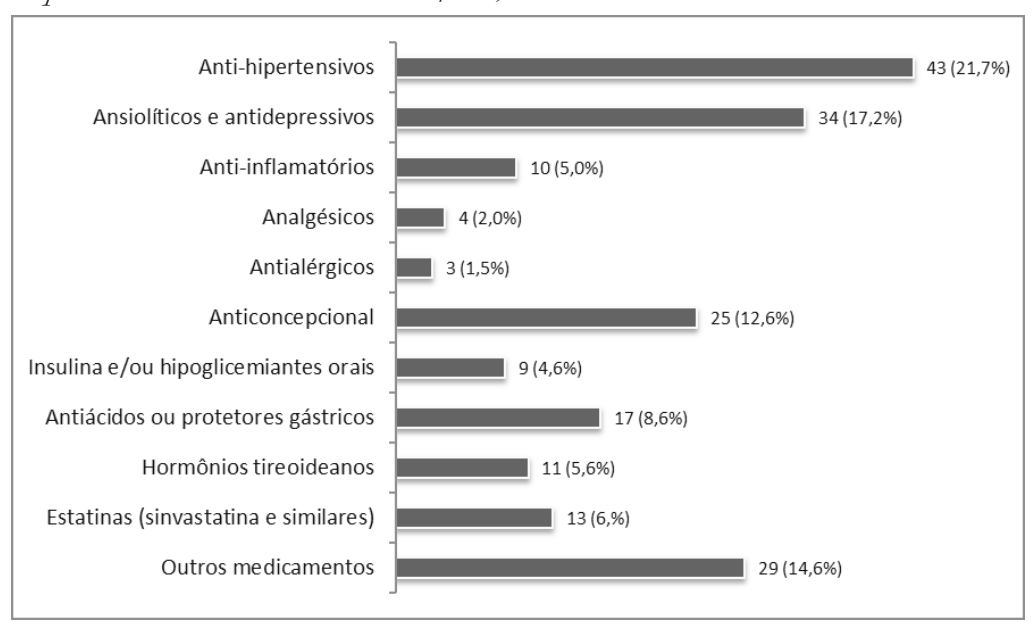

Rev. Bras. Pesq. Saúde, Vitória, 22(4): 104-112, out-dez, 2020 | ISSN: 2175-3946 | DOI: 10.47456/rbps.v22i4.19243 | 107 
Verificou-se que a maioria dos pacientes consumia açúcar regularmente $(69,0 \%)$ e relatou escovar os dentes três vezes ao dia $(63,0 \%)$, de modo semelhante entre os sexos. A frequência do uso do fio dental foi mais prevalente nas mulheres $(71,3 \%)$ que nos homens $(40,0 \%)$, sendo essa diferença significante $(p=0,00)$. Observou-se ainda que o uso do fio dental era mais frequente entre os participantes com mais idade, sendo essa diferença significativamente maior $(\mathrm{p}=0,00)($ Tabela 4$)$.

Tabela 4 - Distribuição dos hábitos de saúde bucal em relação ao sexo e faixa etária dos pacientes atendidos na disciplina de Odontologia Restauradora Clínica da UFES, no periodo de 2010 a 2015. Vitória/ES, Brasil

\begin{tabular}{|c|c|c|c|c|c|c|c|c|c|}
\hline \multirow{3}{*}{ Variáveis } & \multirow{2}{*}{\multicolumn{3}{|c|}{ Pacientes }} & \multicolumn{5}{|c|}{ SEXO } & \multirow{3}{*}{ p-valor } \\
\hline & & & & \multicolumn{2}{|c|}{ Masculino } & \multicolumn{3}{|c|}{ Feminino } & \\
\hline & \multicolumn{3}{|c|}{ n (\%) } & $\mathbf{n}$ & (\%) & $\mathbf{n}$ & \multicolumn{2}{|c|}{$\%$} & \\
\hline \multicolumn{10}{|c|}{ Consome Açúcar/Doce } \\
\hline Sim & 153 & \multicolumn{2}{|c|}{69,0} & 41 & 74,5 & 112 & \multicolumn{2}{|c|}{67,1} & \multirow[t]{2}{*}{$0,30^{*}$} \\
\hline Não & 69 & \multicolumn{2}{|c|}{31,0} & 14 & 25,5 & 55 & \multicolumn{2}{|c|}{32,9} & \\
\hline Total & 222 & \multicolumn{2}{|c|}{100,0} & 55 & 100,0 & 167 & \multicolumn{2}{|c|}{100,0} & \\
\hline \multicolumn{10}{|l|}{ Escovação Diária } \\
\hline Uma vez & \multicolumn{2}{|c|}{11} & 4,9 & 4 & 7,3 & 7 & \multicolumn{2}{|c|}{4,2} & \\
\hline Duas vezes & \multirow{2}{*}{$\begin{array}{c}47 \\
140\end{array}$} & & & 16 & 29,1 & 31 & & & $0,26^{\star \pi}$ \\
\hline Três vezes & & & & 29 & 52,7 & 111 & & & \\
\hline Mais de três vezes & 24 & & & 6 & 10,9 & 18 & & & \\
\hline Total & 22 & & & 38 & 100,0 & 112 & & & \\
\hline Uso do Fio Dental & & & & & & & & & \\
\hline Sim & 14 & & & 22 & $40,0^{A}$ & 119 & & & $0 \cap 0$ ** \\
\hline Não & 32 & & & 15 & $27,3^{A}$ & 17 & & & 0,00 \\
\hline Às vezes & 49 & & & 18 & $32,7^{\mathrm{A}}$ & 31 & & & \\
\hline Total & 22 & & & 55 & 100,0 & 167 & & & \\
\hline & & & & FAI) & A ETÁRIA & & & & \\
\hline Variáveis & 21 & anos & & 40 anos & & 50 anos & & 0 anos & p-valor \\
\hline & $\mathbf{n}$ & $\%$ & $\mathbf{n}$ & $\%$ & $\mathbf{n}$ & $\%$ & $\mathrm{n}$ & $\%$ & \\
\hline Consumo de Açúc & oce & & & & & & & & \\
\hline Sim & 34 & 81,0 & 32 & 64,0 & 28 & 63,6 & 59 & 68,6 & $0,26^{*}$ \\
\hline Não & 08 & 19,0 & 18 & 36,0 & 16 & 36,4 & 27 & 31,4 & \\
\hline Escovação Diária & & & & & & & & & \\
\hline Uma vez & 01 & 2,4 & 4 & 8,0 & 02 & 4,5 & 04 & 4,7 & \\
\hline Duas vezes & 09 & 21,4 & 12 & 24,0 & 05 & 11,4 & 21 & 24,4 & $0,75^{\star *}$ \\
\hline Três vezes & 27 & 64,3 & 28 & 56,0 & 32 & 72,7 & 53 & 61,6 & \\
\hline Mais de três vezes & 05 & 11,9 & 06 & 12,0 & 05 & 11,4 & 08 & 9,3 & \\
\hline Uso do Fio Dental & & & & & & & & & \\
\hline Sim & 17 & $40,5^{\mathrm{A}}$ & 29 & $58,0^{A B}$ & 31 & $70,5^{\mathrm{B}}$ & 64 & $74,4^{\mathrm{BC}}$ & 000 ** \\
\hline Não & 08 & $19,0^{A}$ & 05 & $10,0^{\mathrm{A}}$ & 05 & $11,4^{\mathrm{A}}$ & 14 & $16,3^{\mathrm{A}}$ & 0,00 \\
\hline Às vezes & 17 & $40,5^{\mathrm{A}}$ & 16 & $32,0^{\mathrm{A}}$ & 08 & $18,2^{A B}$ & 08 & $9,3^{\mathrm{B}}$ & \\
\hline Total & 42 & 100,0 & 50 & 100,0 & 44 & 100,0 & 86 & 100,0 & - \\
\hline
\end{tabular}

* Teste exato de Fisher. ** Razão de Máxima Verossimilhança. Diferença significativa para p<0,05. Letras diferentes denotam diferenças estatisticamente significantes entre as proporções. 


\section{DISCUSSÃO|}

No presente estudo, a maioria dos atendimentos clínicos foi realizada em pacientes do sexo feminino $(75,2 \%$ da amostra), confirmando os achados relatados em outros estudos realizados no Brasi ${ }^{14,15}$. Segundo Tortamano et al. ${ }^{16}$, a maior procura por tratamento odontológico pelo sexo feminino ocorre devido ao maior comprometimento das mulheres com a saúde e estética. De modo geral, esse comportamento pode ser explicado por questões culturais e sociais, em que as mulheres normalmente são responsáveis por acompanhar os filhos e os idosos ao médico e frequentar o pré-natal, tornando-as naturalmente mais conscientes da necessidade de cuidar da saúde ${ }^{17}$. Os homens, quando são questionados quanto aos motivos que os levam a não buscar atendimento nas unidades de saúde, alegam, dentre outros fatores, horários inadequados de funcionamento dos serviços que ocorrem durante a jornada de trabalho, além da demora no atendimento ${ }^{18}$.

A década de idade que mais buscou atendimento odontológico situou-se entre 51 e 60 anos $(38,8 \%)$, diferentemente do verificado em outros estudos nos quais a maior procura pelos serviços odontológicos se concentrou entre 20 e 44 anos $^{5,17}$. Já Borghi et al. ${ }^{14}$ constataram uma maior concentração de pacientes $(47,7 \%)$ na faixa etária entre 30 e 49 anos. Um fator colaborador para esse resultado pode ter sido que grande parte dos pacientes acima de 51 anos se encontram aposentados e com maior disponibilidade de tempo para cuidar da saúde. No Brasil, a limitação das ações de saúde bucal para adultos e idosos, grupos historicamente pouco priorizados pelos modelos assistenciais, faz com que suas necessidades de tratamento se acumulem, acarretando perdas dentárias prematuras e grande demanda por tratamentos especializados. As faixas etárias mais jovens vêm sendo atendidas com mais frequência através dos programas de atenção à saúde bucal implantados a partir das diretrizes da Política Nacional de Saúde Bucal quando comparadas com as dos grupos etários mais velhos ${ }^{1,19}$, por exemplo, as ações desenvolvidas pelos dentistas do Programa Saúde na Escola (PSE) que buscam avaliar e promover a saúde bucal dos educandos ${ }^{20}$.

Ao se analisar a procedência demográfica da população estudada, verificou-se que $94,6 \%$ dos pacientes eram moradores dos municípios componentes da Região Metropolitana da Grande Vitória. Destaca-se o fato de que apenas 20,3\% residiam na capital, Vitória, o que pode evidenciar possíveis falhas dos municípios circunvizinhos em oferecer serviços públicos de atendimento odontológico à população. A qualidade do atendimento também é um aspecto a ser considerado. Muitos pacientes preferem ser atendidos nas Universidades, pois confiam na qualidade dos serviços oferecidos ${ }^{4,17}$. Esses resultados são discordantes dos achados de Reis, Santos e Leles ${ }^{7}$ que verificaram que $72,1 \%$ da amostra estudada moravam na capital, e apenas $23,8 \%$ eram da região metropolitana. Outro fator que pode ter contribuído para esse resultado é que na capital, Vitória, residem pessoas de maior poder aquisitivo ao contrário dos municípios vizinhos. De Paula et al. ${ }^{21}$ observaram que o nível socioeconômico baixo das pessoas foi determinante na procura do serviço odontológico do município de Juiz de Fora (MG) e indiferente quanto ao local de residência.

O número de doenças relatadas pelos pacientes foi de $67,6 \%$, sendo significativamente maior na faixa etária de 51 a 60 anos. Esse resultado corrobora os observados por Pinheiro et al. ${ }^{22}$, onde $31,6 \%$ dos pacientes disseram ter pelo menos uma doença crônica, com predomínio de doenças musculoesquelética e cardiovasculares, e maior incidência em pacientes acima de 50 anos. No Brasil, o índice de mortalidade por doenças cardiovasculares é preocupante, seja por alimentação inadequada, tabagismo, baixa atividade física ou consumo de álcool, dentre outros fatores ${ }^{23}$. Neste estudo, os pacientes com doenças cardiovasculares representaram 23,8\% da amostra, seguidos pela presença de doenças infecciosas $(15,4 \%)$, resultado concordante com a pesquisa do Ministério da Saúde que, em 2010, apontou as doenças cerebrovasculares, infarto agudo do miocárdio e a pneumonia como as que mais mataram no Brasil ${ }^{24}$. Ao contrário dos resultados de De Paula et al. ${ }^{21}$, que observaram maior índice de doenças relatadas para alergias e sinusite, sendo a dor de cabeça a maior queixa dos pacientes.

Um parâmetro estaticamente significante observado nesta pesquisa foi o uso regular de medicamentos pelos pacientes que se aproximou de $60 \%$ da população estudada, superior ao de outros estudos que encontraram valores menores de $50 \%{ }^{21}$. O uso de medicamentos por pacientes na faixa etária de 51 a 60 anos foi de 79,1\% ( $\mathrm{p}=0,00)$ pelo fato de que com o avançar da idade aumenta o número de doenças e afecções múltiplas no mesmo indivíduo ${ }^{25}$. Observouse ainda que um maior consumo de medicamentos foi relatado por mulheres, $65,9 \%$ versus $40 \%$ por homens, considerando uma melhor autopercepção de saúde pelo sexo feminino ${ }^{26}$. Os anti-hipertensivos corresponderam a $21,7 \%$, e os ansiolíticos e antidepressivos representaram 
$17,2 \%$ dos medicamentos de uso regular relatados pelos pacientes. Em contrapartida, Bertoldi et al. ${ }^{26}$ observaram que os analgésicos foram mais utilizados $(26,6 \%)$ em seus estudos, seguidos dos medicamentos para problemas cardiovasculares $(24,6 \%)$. O profissional deve possuir conhecimento acerca das alterações fisiológicas do envelhecimento, conhecer possíveis interações entre saúde bucal e saúde geral, saber lidar com possíveis efeitos colaterais dos medicamentos ${ }^{27}$. O registro detalhado nos prontuários das morbidades e medicações de uso regular pelo paciente oferece importantes indicadores para a conduta do cirurgião-dentista.

Em relação aos hábitos de saúde bucal dos pacientes nos prontuários consultados, o consumo de doce/açúcar se mostrou excessivo $(69,0 \%)$ e, desse modo, pode interferir na saúde geral causando doenças como obesidade, diabetes, hipertensão $\operatorname{arterial}^{28}$, além de aumentar o risco à cárie denta ${ }^{10}$ e dor odontológica ${ }^{29}$. A maioria dos pacientes relatou escovar os dentes 3 vezes ao dia, e a frequência diária do uso de fio dental foi significativamente maior nas mulheres e na faixa etária de 51 a 60 anos. Nos estudos de Lisbôa e $\mathrm{Abegg}^{30}$ realizados no Rio Grande do Sul, o uso do fio dental também foi mais frequente em mulheres, no entanto mais predominante na faixa etária de 20 a 34 anos. O cirurgião-dentista como agente de promoção de saúde deve orientar e motivar o paciente para que melhore a sua saúde, fornecendo informações adequadas sobre hábitos de higiene bucal e cuidados diários ${ }^{20}$.

O questionário de saúde geral, quando adotado de modo consciente e com atenção, deixa de ser uma formalidade e passa a ser uma conduta de zelo pela saúde do paciente ${ }^{7}$, A construção do histórico médico do paciente auxilia na prevenção de doenças, garantindo o seu bem-estar e a qualidade de vida e assim favorece o aumento da expectativa de vida ${ }^{27}$. Além disso, o conhecimento dos dados demográficos, do histórico de saúde geral e dos hábitos de saúde bucal dos pacientes registrados nos prontuários são importantes para planejamento de ações acadêmicas visando à melhoria do ensino e à qualidade do serviço prestado à população.

\section{CONCLUSÃO |}

O perfil epidemiológico dos pacientes atendidos na disciplina de ORC/UFES apresentou:
- Predominância do sexo feminino e a faixa etária de 51 a 60 anos;

- Mais de três doenças sistêmicas concomitantes foram relatadas pela maioria dos pacientes, sendo as cardiovasculares as mais frequentes;

- A medicação mais utilizada foi anti-hipertensivos, seguida de antidepressivos e ansiolíticos;

- Quanto aos hábitos de saúde, os pacientes relataram alto consumo de açúcar e escovação dentária 3x/dia. $\mathrm{O}$ uso do fio dental foi predominante em pacientes acima de 51 anos.

\section{REFERÊNCIAS |}

1. Brasil. Ministério da Saúde. Política Nacional de Atenção Básica [Internet]. Brasília: Ministério da Saúde; 2004 [acesso em 03 mar 2016]. Disponível em: http:/ / dab.saude.gov.br/ portaldab/dab.php.

2. Guo Y, Logan HL, Dodd VJ, Muller KE, Marks JG, Riley JL. Health literacy: a pathway to better oral health. Am J Public Health. 2014; 104(7):e85-91.

3. Polverini PJ. A curriculum for the new dental practitioner: preparing dentists for a prospective oral health care environment. Am J Public Health. 2012; 102(2):e1-3.

4. Domingos PSA, Rossato EM, Belline A. Levantamento do perfil social, demográfico e econômico de pacientes atendidos na clínica de Odontologia do Centro Universitário de Araraquara. Revista Brasileira Multidisciplinar. 2014; 17(1):37-50.

5. Nakamura CC, Gonçalves DR, Castro RFM, Closs PS. Perfil dos pacientes atendidos na clínica odontológica da faculdade São Lucas, Porto Velho. Saber Cient Odontol. 2010; 1(1):42-52.

6. Nassri MRG, Silva AS, Yoshida AT. Levantamento do perfil socioeconômico de pacientes atendidos na clínica odontológica da Universidade de Mogi das Cruzes e do tratamento ao qual foram submetidos: clínica endodôntica. RSBO. 2009; 6(3):272-8. 
7. Reis SCGB, Santos LB, Leles CR. Clínica integrada de ensino odontológico: perfil dos usuários e necessidades odontológicas. Rev Odontol Brasil Central. 2011; 20(52):46-51.

8. Sponchiado Júnior EC, Souza TB. Estudo da demanda ambulatorial da clínica de odontologia da Universidade do Estado do Amazonas. Ciênc Saúde Coletiva. 2011; 16(Suppl 1):993-7.

9. Fernandes SKS, Coutinho ACM, Pereira EL. Avaliação do perfil socioeconômico e nível de satisfação dos pacientes atendidos em Clínica Integrada Odontológica Universitária. Rev Bras Promoç Saúde. 2008; 21(2):137-43.

10. Gallarreta FWM, Turssi CP, Palma-Dibb RG, Serra MC. Histórico de saúde: atenção a condições sistêmicas e suas implicações, sobretudo nos fatores de risco de cárie. Rev Odonto Ciênc. 2008; 23(2):192-6.

11. Sparer M. Health Care Reform and the Future of Dentistry. Am J Public Health. 2011; 101(10):1841-4.

12. Habegger B. Strategic foresight in public policy: reviewing the experiences of the UK, Singapore, and the Netherlands. Futures. 2010; 42(1):49-58.

13. Ministério da Educação (Brasil). Resolução $n^{\circ} 3$, de 19 de fevereiro de 2002. Institui as diretrizes curriculares nacionais dos cursos de farmácia e odontologia [Internet]. Diário Oficial da União 7 dez 2001 [acesso em 04 mar 2016]. Disponível em: http://portal.mec.gov.br/cne/ arquivos/pdf/CES1300.pdf.

14. Borghi WMMC, Sundefeld MLMM, Saliba NA, Moimaz SAS, Poi WR. Razões que influenciam o paciente a buscar atendimento odontológico na clínica integrada. Pesq Bras Odontoped Clin Integr. 2008; 8(3):347-52.

15. Pinto RS, Matos DL, Loyola Filho AI. Características associadas ao uso de serviços odontológicos públicos pela população adulta brasileira. Ciênc Saúde Coletiva. 2012; 17(2):531-44.

16. Tortamano IP, Leopoldino VD, Borsatti MA, Penha SS, Buscariolo IA, Costa CG, et al. Aspectos epidemiológicos e sociodemográficos do setor de Urgência da Faculdade de Odontologia da Universidade de São Paulo. Rev Pos Grad. 2007; 13(4):299-306.
17. Sanchez HF, Drumond MM. Atendimento de urgências em uma Faculdade de Odontologia de Minas Gerais: perfil do paciente e resolutividade. Rev Gaúch Odontol (Online). 2011; 59(1):79-86.

18. Bertolini DNP, Simonetti JP. O gênero masculino e os cuidados de saúde: a experiência de homens de um centro de saúde. Esc Anna Nery. 2014; 18(4):722-7.

19. Baldani MH, Brito WH, Lawder JAC, Mendes YBE, Silva FFM, Antunes JLF. Determinantes individuais da utilização de serviços odontológicos por adultos e idosos de baixa renda. Rev Bras Epidemiol. 2010; 13(1):150-62.

20. Brasil. Ministério da Saúde. Ministério da Educação. Programa saúde na escola (passo a passo): tecendo caminhos da intersetorialidade [Internet]. Brasília: Ministério da Saúde/Ministério da Educação; 2011 [acesso em 03 out 2018]. Disponível em: http://189.28.128.100/ dab/docs/legislacao/passo_a_passo_pse.pdf.

21. Paula JS, Oliveira M, Soares MRSP, Chaves MGAM, Mialhe FL. Perfil epidemiológico dos pacientes atendidos no Pronto Atendimento da Faculdade de Odontologia da Universidade Federal de Juiz de Fora. Arq Odontol. 2012; 48(4):257-62.

22. Pinheiro RS, Viacava F, Travassos C, Brito AS. Gênero, morbidade, acesso e utilização de serviços de saúde no Brasil. Ciênc Saúde Coletiva. 2002; 7(4):687-707.

23. Mansur AP, Favarato D. Tendências da taxa de mortalidade por doenças cardiovasculares no Brasil, 19802012. Arq Bras Cardiol. 2016; 107(1):20-5.

24. Previdelli A. As 20 doenças que mais matam no Brasil [Internet]. Exame 2013 jan 24 [acesso em 14 maio 2017]. Disponível em: http://exame.abril.com.br/brasil/as-20doencas-que-mais-matam-no-brasil.

25. Silva AL, Saintrain MVL. Interferência do perfil epidemiológico do idoso na atenção odontológica. Rev Bras Epidemiol. 2006; 9(2):242-50.

26. Bertoldi AD, Barros AJD, Hallal PC, Lima RC. Utilização de medicamentos em adultos: prevalência e determinantes individuais. Rev Saúde Pública. 2004; 38(2):228-38. 
27. Rego MA, Rocha WMS, Ferreira EF. Perfil do paciente idoso referenciado ao consultório odontológico do Instituto Jenny de Andrade Faria HC/UFMG. Rev Odontol UNESP. 2013; 42(1):42-7.

28. Davoglio RS, Aerts DRGC, Abegg C, Freddo SL, Monteiro L. Fatores associados a hábitos de saúde bucal e utilização de serviços odontológicos entre adolescentes. Cad Saúde Pública. 2009; 25(3):655-67.

29. Knackfuss AP, Costenaro RGS, Zanatta FB. Dor odontológica e indicadores de risco em jovens. Rev Gaúch Odontol (Online). 2011; 59(2):185-91.

30. Lisbôa IC, Abegg C. Hábitos de higiene bucal e uso de serviços odontológicos de adolescentes e adultos do Município de Canoas, RS, Brasil. Epidemiol Serv Saúde. 2006; 15(4):29-39.

Correspondência para/ Reprint request to:

Martha Chiabai Cupertino de Castro

Av. Marechal Campos, 1468,

Maruipe, Vitória/ES, Brasil

CEP 29.040-090

E-mail:marthachiabai@gmail.com

Recebido em: 14/03/2018

Aceito em: 05/03/2021 